\title{
FIRE DETECTION IN VIDEO STREAM BY USING SIMPLE ARTIFICIAL NEURAL NETWORK
}

\author{
Peter Jankůํ, Zuzana Komínková Oplatková ${ }^{1}$, Tomáš Dulík ${ }^{1}$, Petr Snopek ${ }^{2}$, Jiří Líba ${ }^{2}$ \\ ${ }^{1}$ Tomas Bata University in Zlin \\ Faculty of Applied Informatics \\ Nam. T.G. Masaryka 5555, 76001 Zlin, Czech Republic \\ janku@utb.cz,oplatkova@utb.cz,dulik@utb.cz \\ ${ }^{2}$ UNIS a.s. \\ Jundrovska 33, 62400 Brno, Czech Republic \\ psnopek@unis.cz,jliba@unis.cz
}

\begin{abstract}
This paper deals with the preliminary research of the fire detection in a video stream. Early fire detection can save lives and properties from huge losses and damages. Therefore the surveillance of the areas is necessary. Early fire discovery with high accuracy, i.e. a low number of false positive or false negative cases, is essential in any environment, especially in places with the high motion of people. The traditional fire detection sensors have some drawbacks: they need separate systems and infrastructure to be implemented, to use sensors in the case of the industrial environment with open fire technologies is often impossible, and others. The fire detection in a video stream is one of the possible and feasible solutions suitable for replacement or supplement of conventional fire detection sensors without a need for installation a huge infrastructure. The paper provides the state of the art in the fire detection. The following part of the paper proposes the new system of feature extraction and describes the feedforward neural network which was used for the training and testing of the proposed idea. The promising results are presented with over 93\% accuracy on a selected dataset of movies which consist of more and highly varied instances than published by other researchers involved in the fire detection field. The structure of the neural networks promises higher computational speed than currently implemented deep learning systems.
\end{abstract}

Keywords: Fire detection, Computer vision, Artificial neural networks

\section{Introduction}

This research introduced in this paper deals with early fire detection in a video stream. The method can serve as a replacement or supplement of conventional fire detection sensors without a need for installation a huge infrastructure. Early fire detection can save lives and properties from huge losses and damages. Therefore the surveillance of important areas is necessary. Currently, the surveillance systems are installed almost anywhere since the price for the CCTV (closedcircuit television) decrease within the time and is affordable from small traders through integrated rescue system to huge international corporates. Nowadays, decreasing the price of camera systems together with the increasing of computer and computational power play into the hands of the progressive development of research in the area of the computer vision systems.

Therefore, the proposed method implements computer vision technologies with machine learning techniques to help to discover the fire as earliest as possible to be able to react and solve the situation. The price, installation and usage supposed to be lower and simpler compared to the traditional fire detection sensors.

The traditional fire detection sensors have some drawbacks. They usually need separate systems and infrastructure to be implemented. The usage of these sensors is often impossible in the case of the industrial environment with open fire technologies. The smoke from the standard production (e.g. during welding) will not be distinguished from the real dangerous fire by these types of sensor, and other drawbacks could follow. The fire detection in a video stream is one of the possible and feasible solutions suitable for replacement or supplement of conventional fire detection sensors without a need for installation a huge infrastructure. The proposed system will consist of the supplementary computational system to check the video stream and highlight the suspicious area with fire.

The popularity of computer vision in the fire detection could be illustrated by the quantity of published papers in scientific databases. For example, about 200 results are provided by searching the keywords "fire detection computer vision" in the IEEE database. Almost the quarter of this amount has been published during the last two years (2017 2018). The visual appearance of fire is usually composed of different parts. Smoke and flame contours belong to primary classification groups of visual fire discovery. Separately or their combination is an essential part of fire detection algorithms. The following subsections will be aimed at smoke detection, color detection and movement detection which will determine later if the fire is present in the video stream or not. Besides the mentioned subsections, intelligent classifiers such as artificial neural networks, specifically deep learning systems, convolutional systems, are employed by researchers to help with the detection. The proposed system uses a classical shallow artificial neural network of feedforward type with Levenberg-Marquardt training algorithm to obtain an as fast and simple solution as possible. 


\subsection{Smoke Detection}

Some published algorithms use only smoke areas for early-fire detection. These algorithms usually work with a smoke color (the grey color is supposed for smoke) and a smoke movement (upward direction is supposed). Figure 1 depicts the results of the described algorithm in [1].

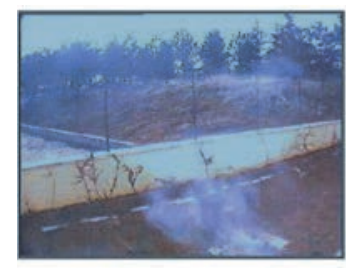

(a)

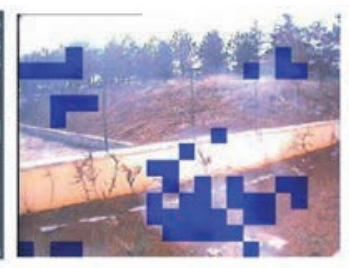

(b)

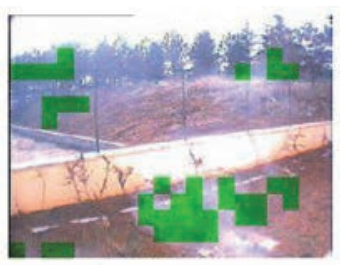

(c)

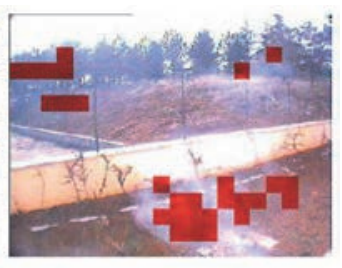

(d)

Figure 1: Smoke detection algorithm. a) original picture, b) movement detection, c) color detection, d) final decision [1]

The higher complex algorithm of visual smoke recognition is published by Tripathi et al. in [2]. The amount of energy in picture foreground and background together with the level of blur in smoke areas were appended into final detection decision.

The usability of the smoke detection algorithm is limited in environments where fire or fire-like action are part of standard processes. The detection ability decreases especially in industrial buildings where the smoke originator can be found for instance during welding. Therefore, this research is aimed at methods for fire-flame detection.

\subsection{Color Detection}

Fire-flame computer vision analysis could also be focused on different visual aspects. The fire-flame color is used as the first marker in a significant amount of published studies. Even if new color space was published by Khatami et al. [3, 4], many algorithms use simplified color rules. Their work [3, 4] was aimed to develop a method for fire clustering by color. Swarm algorithms were used for defining coefficients of the convolutional matrix in order to select fire-like areas. More straightforward access for fire color classification based on the RGB color model is used as an essential step in other published algorithms $[5,6]$. In these researches, the simple equation (1) is applied together with thresholds to different color parts.

$$
R>G>B
$$

Even if the RGB color model is the most straightforward in computer vision, it is not the only one used for fire-color detection. Among other color models, following three models belong: the YUV in a study published by Li et al. [7], HSV model used by Vijayalakshmi et al. in [8] and YCbCr one used by Ye et al. in [9].

Even though color detection is widely used as a first step in published algorithms, another step usually follows (motion detection, optical flow, artificial neural network detection and analysis).

\subsection{Movement Detection}

A movement can be represented by a various set of parameters in pictures based on computer vision techniques. The algorithm described by Wattanachote et al. [10], the optical flow method is applied to the series of images in order to achieve motion vectors. The demonstration of motion vector fields is represented in Figure 2.

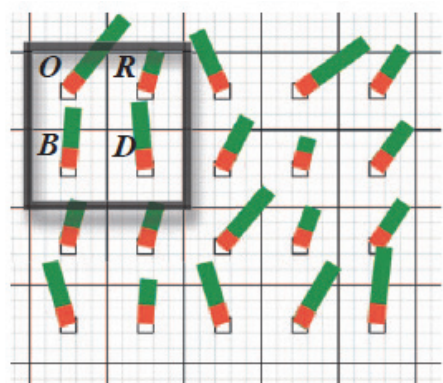

(a) Motion vector field, which the vectors were constructed by $5 \times 5$ pixels SB (SB5).

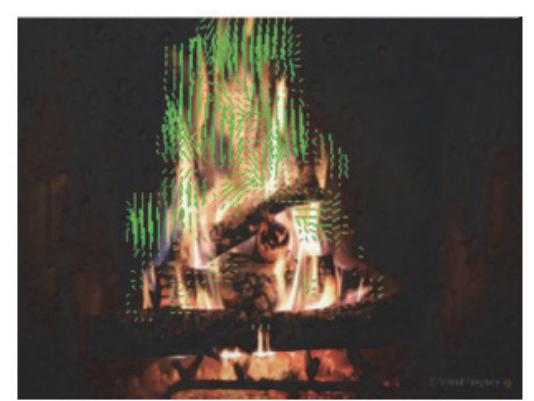

(b) Dynamic fire texture and its motion vector field, each vector was created by $5 \times 5$ pixels $\mathrm{SB}$ (SB5).

Figure 2: Demonstration of motion vector fields resulted by Farneback's method [33][10]. 
Well cited algorithm is described by Töreyin et al. [11]. Besides another feature, the Wavelet analysis is used to investigate the input video stream. Authors are focused on moving color areas in the picture as well as on changing of the file-flame shape. The similarity of flame shape and its variations are used by another research [5] as one of the fire detection decision aspects. In the study published by Abdullahi et al. [6], the picture movement is computed in two steps. In the first step, the simple difference between consecutive frames is carried out. In a further step, the distance change of contour points from the precalculated center is observed.

\subsection{Usage of Artificial Neural Networks}

Except for the above-described techniques, machine learning approaches are adopted into the field of fire detection since the variability of fire-flames is huge and an intelligent detection system is an advantage. High-quality classifiers are represented by artificial neural networks. As described in research published by Mao et al. [12], the using of neural networks in computer vision could be based on two ways. The elementary way of the first approach is to extract some markers/features from the image and afterward to make a decision about fire by using a simple shallow artificial neural network. This approach could bring a faster algorithm. On the other hand, the success rate is strictly influenced by the method of feature extraction.

The most popular approach is based on methods of deep learning algorithms and convolutional neural networks in these days [13-17]. This approach usually requires no feature extraction, but the final neural networks are significantly more complex and more computational resources are requested.

This paper will consider the usage of shallow artificial neural networks based on feedforward type with LevenbergMarquardt training algorithm to speed up the computation.

\section{Artificial Neural Network}

Artificial neural networks (ANN) are inspired by the biological neural nets and are used for complex and difficult tasks [18-23]. The most often usage is a classification of objects. Many tasks can be transferred and can be recognized as a classification task as also in this case. The aim of the paper is to pick a suspicious area in a video stream and classify if the fire is present there or not. ANNs are capable of generalization and hence the classification is natural for them. Many other applications are spread in all fields of the human activities - from simple to very complex tasks: pattern recognition, control, filtering of signals, data approximation, natural language processing, image processing, cryptography and steganography and other security areas.

Currently, ANNs are divided into two main groups - shallow nets and deep learning systems (mainly with convolutional neural networks). If image processing is considered, mostly convolutional neural networks are employed because they respect the spatial layout of the image itself. It is not necessary to look for the suitable feature selection method since it is included in the principles of the training algorithm.

Compared to that, first approaches in the neural networks can be considered as shallow nets. These include feedforward net with supervision which is used in the simulations and testing of the proposed solution of fire detection in a video stream. The main reason was to find as minimalistic structure as possible. The assumption is to reduce the speed in a supplemental system to existing surveillance camera systems which will process the video stream and highlight the suspicious areas with fire.

ANN needs a training set of known solutions to be trained on them. Supervised ANN has to have inputs and also required output(s). Therefore, the labeled data were necessary to collect.

The neural network works so that properties or attributes of instances in the training sets are transferred into suitable input values, i.e. into the vector of selected features. These inputs are multiplied by weights which are adjusted and optimized during the training to produce an inner potential inside the node. In the node, the sum of inputs multiplied by weights is transferred through a mathematical function like sigmoid, linear function or hyperbolic tangent etc. The output then represents the class of the object. In this case, just one output node is required - with the output 0 (no fire) or output equal to 1 (fire is present in the image or relevant part of the image).

\section{Problem Formulation}

As described in previous sections, the fire-detection by using computer vision is a highly-studied scientific field. Although a large number of algorithms were published, the set of weaknesses is still present - low success rate limited by used scene and/or high computation demands.

This preliminary study tries to find an algorithm which would be able to perform early fire detection in a video stream with special attention on computation intense. The algorithm should be able to work with different video sources, many video streams at one moment, different video formats and resolutions. The system will serve as a supportive tool which could be deployed into existing systems of surveillance cameras.

\section{The Basic Algorithm Workflow}

The algorithm workflow represents the Figure 3. As can be seen, the algorithm works in consecutive steps. As the input into the algorithm, the frame from a video stream is used. The output of the algorithm represents the decision whether or not the fire is present in the video stream. 
In the first two steps of the algorithm, the input frame is investigated in order to get information about movement in consecutive frames and fire-like color areas. Based on these steps, the suspicious areas are selected, grouped by specified rules. These groups are tracked in time by using special data container. After these steps, the first set of pre-decisions are made. If the suspicious area is confirmed, the set of features is extracted from histogram values. The last step is to provide a final decision based on a simple shallow artificial neural network.

\begin{tabular}{|l|l|}
\hline & - Color detection by using HSL color model \\
\hline & -Movement detection by subtracting frames \\
\hline & -Tracking of suspicious areas in time \\
\hline & - Extracting features from histogram values \\
\hline
\end{tabular}

Figure 3: Definition of algorithm structure

\section{Data}

The data for the experiment were collected from the different sources, different types of cameras, different resolution to secure the generalization of the solution. The dataset consists of 2725 samples for the fire and 4208 negative samples. The videos were taken from varied environments, scenes, colors - as sunset, dancing people in yellow t-shirts in the negative cases with no fire present in the scene. The cases with fire contain instances from building or cars being in flames or welding in the industrial environment and others. Each video was investigated based on the algorithm flow in Figure 3. The total number of input features was only 45 . Examples of extracted features are depicted in Figure 4 . Each chart contains values of the particular feature for all instances in the dataset - left side negative - no fire present, right side positive - with fire. As can be visible, some feature (a) and b)) are more helpful for the decision process, some not (c) and d)). Since this paper deals with preliminary results, the future plans are to decrease the number of features based on the analysis of the impact on the decision process.

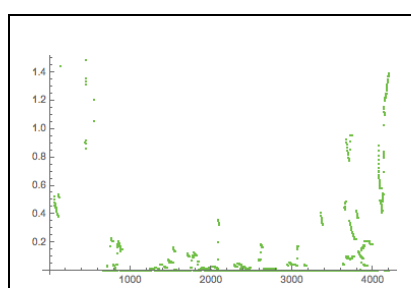

a)

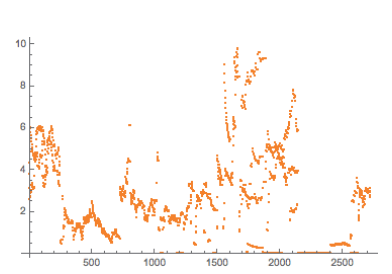

b)

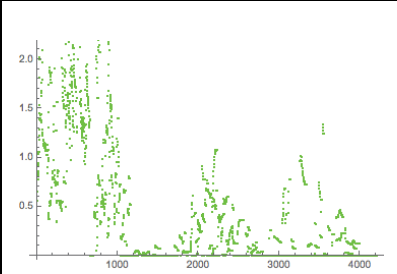

c)

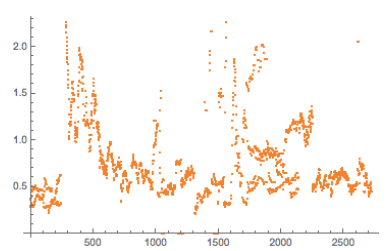

d)

Figure 4: Example of extracted features - a) feature 15 for all negative instances, b) feature 15 for all positive instances with fire, c) feature 42 for all negative instances, d) feature 42 for all positive instances with fire

\section{Results}

The experiments were performed with feedforward artificial neural network and Levenberg-Marquardt training algorithm, with different settings of the hidden nodes (number of nodes from one to twenty) and the combination of the transfer functions were logistic sigmoid, hyperbolic tangent, saturated linear for both hidden nodes and the output node. Following tables shows the example of the best-performed structure -3 hidden nodes, logistic sigmoid function in hidden nodes and hyperbolic tangent in the output node. The output node decides if the fire is present in the particular investigated area or not. Tables 1 and 2 shows the results of the confusion matrix and false positive rate, false negative rate, sensitivity, specificity, accuracy, precision and F1 score for the training and testing. The dataset was imbalanced therefore also a detailed analysis of particular parts (dancing people, fire in a school building, etc.) are given in Tables 3 (training) and 4 (testing). The results show small overfitting. However, the accuracy rate is quite high compared the other published papers.

Table 1: Results for the best found structure - training - 3 hidden nodes, sigmoid transfer function in hidden nodes, hyperbolic tangent in an output node

\begin{tabular}{|crrrr|}
\hline & Training confusion matrix & - 3SigmoidTanh.txt & & N \\
& & P & & \\
True predicted & TP & 4208 & FP & 2719 \\
False predicted & FP & 0 & TN & 99.7798 \\
& TPR & 100. & TNR & 0.220183 \\
\hline Accuracy & FPR & 0. & FNR & \\
Precision & 99.9135 & & & \\
F1 score & 100. & & & \\
TPR-Sensitivity & TNR-Specificity & FPR-False positive rate & FNR-False negative rate \\
\hline
\end{tabular}


Table 2: Results for the best found structure - testing - 3 hidden nodes, sigmoid transfer function in hidden nodes, hyperbolic tangent in an output node

\begin{tabular}{|crrrr|}
\hline & Testing confusion matrix & - 3SigmoidTanh.txt & \\
True predicted & & $\mathrm{P}$ & $\mathrm{N}$ \\
False predicted & TP & 4036 & FP & 282 \\
& FP & 169 & TN & 2438 \\
& TPR & 95.981 & TNR & 89.6324 \\
& FPR & 4.01616 & FNR & 10.3486 \\
\hline Accuracy & 93.4874 & & & \\
Precision & 95.981 & & & \\
F1 score & 94.7084 & & & \\
TPR-Sensitivity & TNR-Specificity & FPR-False positive rate & FNR-False negative rate \\
\hline
\end{tabular}

Table 3: Detailed results for the best found structure - training - 3 hidden nodes, sigmoid transfer function in hidden nodes, hyperbolic tangent in an output node

\begin{tabular}{|c|c|c|c|c|}
\hline \multicolumn{5}{|c|}{ Training accuracy - particular classes - 3SigmoidTanh.txt } \\
\hline Class name & TP in the class & $\begin{array}{l}\text { Total in } \\
\text { the class }\end{array}$ & Error & Accuracy \\
\hline twoManNegtrain & 664 & 664 & $\theta$. & 100. \\
\hline sunsetNegtrain & 46 & 46 & $\theta$. & 100. \\
\hline dancingPeopleNegtrain & 196 & 196 & $\theta$. & 100. \\
\hline dancingGroupNegtrain & 138 & 138 & $\Theta$. & 100. \\
\hline danceNegtrain & 3025 & 3025 & ๑. & 100. \\
\hline coolerbotNegtrain & 139 & 139 & ๑. & 100. \\
\hline schoolpostrain & 246 & 246 & ๑. & 100. \\
\hline roomProblematickyPostrain & 330 & 330 & $\theta$. & 100. \\
\hline house13Postrain & 147 & 147 & ๑. & 100 . \\
\hline carPostrain & 1422 & 1422 & $\odot$. & 100. \\
\hline brushPostrain & 110 & 110 & $\Theta$. & 100. \\
\hline brushBigPostrain & 148 & 148 & ๑. & 100. \\
\hline bonfire 2 Postrain & 160 & 160 & $\theta$. & 100. \\
\hline bonfireMezitrain & 156 & 162 & 3.7037 & 96.2963 \\
\hline
\end{tabular}

Table 4: Detailed results for the best found structure - testing - 3 hidden nodes, simgoid transfer function in hidden nodes, hyperbolic tangent in an output node

\begin{tabular}{|c|c|c|c|c|}
\hline \multicolumn{5}{|c|}{ Testing accuracy - particular classes - 3SigmoidTanh.txt } \\
\hline Class name & TP in the class & $\begin{array}{l}\text { Total in } \\
\text { the class }\end{array}$ & Error & Accuracy \\
\hline twoManNegtest & 664 & 664 & $\theta$. & 100. \\
\hline sunsetNegtest & 45 & 45 & $\theta$. & 100. \\
\hline dancingPeopleNegtest & 160 & 196 & 18.3673 & 81.6327 \\
\hline dancingGroupNegtest & 105 & 138 & 23.913 & 76.087 \\
\hline danceNegtest & 2938 & 3024 & 2.84392 & 97.1561 \\
\hline coolerbotNegtest & 124 & 138 & 10.1449 & 89.8551 \\
\hline schoolpostest & 210 & 245 & 14.2857 & 85.7143 \\
\hline roomProblematickyPostest & 327 & 329 & 0.607903 & 99.3921 \\
\hline house13Postest & 146 & 146 & ๑. & 100. \\
\hline carPostest & 1220 & 1422 & 14.2053 & 85.7947 \\
\hline brushPostest & 108 & 110 & 1.81818 & 98.1818 \\
\hline brushBigPostest & 147 & 147 & $\theta$. & 100. \\
\hline bonfire 2 Postest & 160 & 160 & $\theta$. & 100. \\
\hline bonfireMezitest & 120 & 161 & 25.4658 & 74.5342 \\
\hline
\end{tabular}

In the area of computer vision systems, three parameters could be used for comparison of algorithm sensitivity - accuracy, false positive rate, false negative rate. In spite of these parameters are well known and often used, comparing of algorithms is not an easy task. The main problem lies in the used dataset - if the dataset is changed, these monitored parameters are changed too. Moreover, most of the published papers using really week dataset with only a few videos as input. Usable comparison of algorithm success could be found in [7]. Set of different algorithms is compared on one dataset. Values of algorithm accuracy alter between $61.01 \%$ to $92.30 \%$ as well as false positive range from $8.33 \%$ to $51.67 \%$ and false negative range from $0 \%$ to $24.24 \%$. Even the same dataset was not used in this paper, our results are significantly better. In future work, to use the same dataset for mutual comparison with our proposed algorithm is planned.

\section{Conclusion}

The paper deals with the early fire detection in a video stream. The paper proposes a novelty method with consecutive steps where the final stage is the decision based on a simple shallow artificial neural network. The suggested system is easy to implement to the existed surveillance camera systems for the monitoring of important areas. No reconstructions to build up a new infrastructure is necessary as it would be in the case of traditional sensors for smoke detection. Because of the different scenarios, environments, intelligent systems of detection which will be able to distinguish between the real fire and the standard production processes (e.g. in the case of welding) are necessary to use. The proposed system offers such a detection system with a simple shallow feedforward artificial neural network. Based on the results, it can be stated that the architecture with 3 hidden nodes carried out accuracy almost $100 \%$ for training and over $93 \%$ for testing which form a small overfitting problem but the accuracy threshold is still higher than in other published papers. 
The false positive rate is also low compared to other literature results which means that such a system will not produce false alerts for the emergency. It can be stated, that such a system will help to protect human properties and lives from huge losses and damages in the unmanaged fire attacks. The future plans are to extend simulations with the focus on the overfitting avoidance.

Acknowledgement: This work was supported by Technology Agency of the Czech Republic (TAČR) within the Visual Computing Competence Center - V3C project No. TE01020415, further by the Ministry of Education, Youth and Sports of the Czech Republic within the National Sustainability Programme Project no. LO1303 (MSMT-7778/2014) and by the European Regional Development Fund under the Project CEBIA-Tech no. CZ.1.05/2.1.00/03.0089. This work is also based upon support by COST (European Cooperation in Science \& Technology) under Action IC1406, High-Performance Modelling and Simulation for Big Data Applications (cHiPSet). Last but not least, the work was supported by resources of A.I.Lab at the Faculty of Applied Informatics, Tomas Bata University in Zlin (ailab.fai.utb.cz).

\section{References}

[1] Ha, C., Hwang, U., Jeon, G., Cho, J., Jeong, J.: Vision-Based Fire Detection Algorithm Using Optical Flow. In: 2012 Sixth International Conference on Complex, Intelligent, and Software Intensive Systems. pp. 526-530 (2012).

[2] Tripathi, A.K., Swarup, S.: Visual smoke detection. Lect. Notes Comput. Sci. Subser. Lect. Notes Artif. Intell. Lect. Notes Bioinforma. 10116 LNCS, 128-142 (2017).

[3] Khatami, A., Mirghasemi, S., Khosravi, A., Nahavandi, S.: A New Color Space Based on K-Medoids Clustering for Fire Detection. In: 2015 IEEE International Conference on Systems, Man, and Cybernetics. pp. 2755-2760 (2015).

[4] Khatami, A., Mirghasemi, S., Khosravi, A., Nahavandi, S.: An efficient hybrid algorithm for fire flame detection. In: 2015 International Joint Conference on Neural Networks (IJCNN). pp. 1-6 (2015).

[5] Wang, T., Shi, L., Yuan, P., Bu, L., Hou, X.: A new fire detection method based on flame color dispersion and similarity in consecutive frames. In: 2017 Chinese Automation Congress (CAC). pp. 151-156 (2017).

[6] Abdullahi, Z.S., Dalhatu, S.H., Abdullahi, Z.H.: Development of Fire Detection Algorithm at Its Early Stage Using Fire Colour and Shape Information. IOP Conf. Ser. Mater. Sci. Eng. 344, 012021 (2018).

[7] Li, S., Liu, W., Ma, H., Fu, H.: Multi-attribute based fire detection in diverse surveillance videos. Lect. Notes Comput. Sci. Subser. Lect. Notes Artif. Intell. Lect. Notes Bioinforma. 10132 LNCS, 238-250 (2017).

[8] Vijayalakshmi, S.R., Muruganand, S.: Fire alarm based on spatial temporal analysis of fire in video. In: 2018 2nd International Conference on Inventive Systems and Control (ICISC). pp. 104-109 (2018).

[9] Ye, S., Bai, Z., Chen, H., Bohush, R., Ablameyko, S.: An effective algorithm to detect both smoke and flame using color and wavelet analysis. Pattern Recognit. Image Anal. 27, 131-138 (2017).

[10] Wattanachote, K., Li, L., Lin, Z., Wang, G., Jiang, M., Liu, W.: An investigation on motion periodic series for fire and smoke textures characteristic identification. In: 2017 10th International Conference on Ubi-media Computing and Workshops (Ubi-Media). pp. 1-6 (2017).

[11] Töreyin, B.U., Dedeoğlu, Y., Güdükbay, U., Çetin, A.E.: Computer vision based method for real-time fire and flame detection. Pattern Recognit. Lett. 27, 49-58 (2006).

[12] Mao, W., Wang, W., Dou, Z., Li, Y.: Fire Recognition Based On Multi-Channel Convolutional Neural Network. Fire Technol. 54, 531-554 (2018).

[13] Zhong, Z., Wang, M., Shi, Y., Gao, W.: A convolutional neural network-based flame detection method in video sequence. Signal Image Video Process. 12, 1619-1627 (2018).

[14] Frizzi, S., Kaabi, R., Bouchouicha, M., Ginoux, J., Moreau, E., Fnaiech, F.: Convolutional neural network for video fire and smoke detection. In: IECON 2016 - 42nd Annual Conference of the IEEE Industrial Electronics Society. pp. 877-882 (2016).

[15] Muhammad, K., Ahmad, J., Mehmood, I., Rho, S., Baik, S.W.: Convolutional Neural Networks Based Fire Detection in Surveillance Videos. IEEE Access. 6, 18174-18183 (2018).

[16] Hüttner, V., Steffens, C.R., Botelho, S.S. da C.: First response fire combat: Deep leaning based visible fire detection. In: 2017 Latin American Robotics Symposium (LARS) and 2017 Brazilian Symposium on Robotics (SBR). pp. 16 (2017).

[17] Shen, D., Chen, X., Nguyen, M., Yan, W.Q.: Flame detection using deep learning. In: 2018 4th International Conference on Control, Automation and Robotics (ICCAR). pp. 416-420 (2018).

[18] Hertz, J., Krogh, A., Palmer, R.G.: Introduction to the theory of neural computation. Addison-Wesley/Addison Wesley Longman, Reading, MA, US, ISBN 978-0-201-50395-1 (1991).

[19] Wasserman, P.D.: Neural Computing: Theory and Practice. Coriolis Group, New York, ISBN 978-0-442-20743-4 (1989).

[20] Fausett, L.V.: Fundamentals of Neural Networks: Architectures, Algorithms And Applications. Pearson, Englewood Cliffs, NJ, ISBN 978-0-13-334186-7 (1993).

[21] Jarusek, R., Volna, E., Kotyrba, M.: Robust steganographic method based on unconventional approach of neural networks. Appl. Soft Comput. 67, 505-518 (2018).

[22] Rosebrock, A.: Deep learning for computer vision with Python. PyImageSearch, ISBN 978-1-986538-13-8.

[23] Stanford University CS231n: Convolutional Neural Networks for Visual Recognition, http://cs231n.stanford.edu/. 